\title{
Building an institutional base for Computational Neuroscience: the CBI at UTSA/UTHSCSA
}

\author{
Zhiwei Wang ${ }^{1}$, Kay Robbins ${ }^{2}$, Yufeng Wang ${ }^{3}$, Carolina Livi ${ }^{5}$, Alan D Coop ${ }^{4}$, Fidel Santamaria ${ }^{3}$, Carola Wenk ${ }^{2}$, \\ James $M$ Bower ${ }^{4^{*}}$ \\ From Nineteenth Annual Computational Neuroscience Meeting: CNS*2010 \\ San Antonio, TX, USA. 24-30 July 2010
}

In the Spring of 2005, The University of Texas, San Antonio established the Computational Biology Initiative (CBI) as a mechanism to introduce and then support the local use of computational tools for research and education. In 2006, the CBI expanded to include researchers and educators at the University of Texas Health Science Center, San Antonio. Built on a novel mechanism of computational liaisons, and with a significant focus on education and user support, the CBI has grown to include over 300 registered users, serving 71 individual research laboratories and multiple undergraduate and graduate courses.

\section{Methods}

The CBI core facility at UTSA is built around a High Performance Computing System, a high throughput and high availability data storage system, and a number of high-end workstations. The CBI also supports a number of computational software packages including MatLab, Mathematica, Imaris, Genespring, GENESIS, etc. The CBI is also fully integrated with other core facilities including a Research Imaging Core and a Proteonimics core. In addition to providing resources, however, the CBI from the outset has focused on educating undergraduates, graduate students, postdoctoral fellows and faculty in the use of computational techniques. In that capacity the CBI has sponsored 17 training workshops with more than 400 participants to date. A novel initial feature of the CBI involved the hiring of computational liaisons to serve as the interface with individual laboratories and research projects. These liaisons were Ph.D. research biologists who had also been trained in computation. As trained biologists, they understand the nature of biological experimentation, while their computational training allowed them to infuse computational approaches into existing research projects.

\section{Results}

At present, the CBI has over 300 individual registered users. The CBI currently supports grants totaling $\$ 23,800,000$. Reflecting the extent to which the CBI has now become a core component of research, the CBI director position was recently approved as a State Funding Line. The CBI was also recently awarded the HUB Advocate Department Award for 2009.

\section{Conclusions}

The CBI represents a novel, but successful approach to establishing and then supporting the increased use of computational techniques in biological research broadly defined within a university setting.

\section{Acknowledgements}

Research supported by RCMI (\$1.6 M) and the UT System (\$2.5M)

\section{Author details}

${ }^{1}$ Computational Biology Initiative, UTSA, USA. ${ }^{2}$ Department of Computer Science, UTSA, USA. ${ }^{3}$ Department of Biology, UTSA, USA. ${ }^{4}$ Research Imaging Institute, University of Texas Health Sciences Center at San Antonio, San Antonio, TX 78229, USA. ${ }^{5}$ Department of Nuclear Medicine, University of Texas Health Sciences Center at San Antonio, San Antonio, TX 78229, USA.

Published: 20 July 2010

doi:10.1186/1471-2202-11-S1-P67

Cite this article as: Wang et al:: Building an institutional base for

Computational Neuroscience: the CBI at UTSA/UTHSCSA. BMC

Neuroscience 2010 11(Suppl 1):P67.

\footnotetext{
* Correspondence: bower@uthscsa.edu

${ }^{4}$ Research Imaging Institute, University of Texas Health Sciences Center at San Antonio, San Antonio, TX 78229, USA
} 